\title{
Der Einfluß des neuen Seerechts auf die Meereszonen und die Fischereipolitik Kubas
}

\author{
Von Beate Ratter
}

\section{Einleitung}

Im Dezember 1982 begann, nach Abschluß der zähen Verhandlungen über eine neue UNSeerechtskonvention, die Unterzeichnung der United Nations Law of the Sea Convention in Montego Bay, Jamaica (SRK). Innerhalb der festgesetzten Zeit von zwei Jahren unterzeichneten 159 Staaten der Erde diese neuen Bestimmungen zur Regelung nationaler und internationaler Seerechtsfragen. Bis zum Inkraftreten der Konvention jedoch werden nach Ansicht der Experten noch mehr als 10 Jahre vergehen, denn erst ein Jahr nach der 60. Ratifizierung1 durch einen Unterzeichnerstaat tritt diese Konvention in Kraft (Art. 308 I SRK). Dennoch ist die Wirkung dieser neuen internationalen Richtlinien für die Abgrenzung des Meeresraums auf die nationale Politik der einzelnen Staaten schon heute größer als vielfach angenommen.

Bereits vor Abschluß der Verhandlungen, die sich seit ihrem Beginn 1973 über annähernd 10 Jahre hinzogen, warf die neue Konvention ihre Schatten auf die nationale Gesetzgebung zu Ansprüchen auf den Meeresraum. Durch die neuen Bestimmungen über die Ausdehnung des Küstenmeeres von früher $3 \mathrm{sm}$ auf maximal $12 \mathrm{sm}^{2}$ (Art. 3 SRK), die Beibehaltung der sog. Anschlußzone von nunmehr weiteren $12 \mathrm{sm}$ mit funktional begrenzten Hoheitsrechten (Art. 33 SRK), durch die Einführung der $200 \mathrm{sm}$ breiten ausschließlichen Wirtschaftszone (Exclusive Economic Zone, kurz EEZ, Art. 55 ff. SRK), die dem Staat die exklusiven Rechte über die wirtschaftliche Nutzung der lebenden und nicht lebenden Ressourcen im und auf dem Meeresboden und der Wassersäule zuspricht, sowie detaillierte Vorgaben zur Regelung des intermationalen Seeverkehrs, zum Schutz und zur Erhaltung der maritimen Umwelt und zum Abbau mineralischer Rohstoffe im Bereich der nichtstaatlichen Hohen See, wurde ein entscheidender Schritt hin zur "Territorialisierung des Meeres" unternommen. Diese Entwicklung in Richtung auf eine zunehmende Verstaatlichung des Meeres

1 Bei Ende der Zeichnungsfrist am 9.12.1984 hatten von den 159 Unterzeichnerstaaten bereits 14 Staaten die Konvention ratifiziert. Bis zum Januar 1988 lag die Zahl der Ratifizierungen jedoch nur bei 35. Die Bundesrepublik Deutschland hat neben Großbritannien und den USA die Konvention nicht gezeichnet.

2 Eine Seemeile, $1 \mathrm{sm}$, entspricht der Länge einer Bogenminute auf dem Äquator = 1,852 km; $12 \mathrm{sm}$ $=22,224 \mathrm{~km}$. 
wirkt sich jedoch nicht allein bei der Ausdehnung nationaler Zuständigkeitsbereiche aus, sondern sie besitzt auch intensiven Einfluß auf die nationale Wirtschaftspolitik.

Im Rahmen dieses Beitrages soll an der Territorial- und Fischereipolitik Kubas näher untersucht werden, welche Veränderungen diese noch nicht in Kraft getretene Konvention bereits im voraus bewirkt hat.

\section{Die Entwicklung kubanischer Grenzziehung}

Ein Blick auf die historische Territorialentwicklung Kubas zeigh daß sich die kubanische Grenzziehung mit Ausnahme des US-Militärstützpunktes Guantánamo immer im Meeresraum vollzogen hat. Im Einklang mit der europäischen Praxis der letzten Jahrhunderte, eine $3 \mathrm{sm}$ breite Verteidigungszone als Küstenmeer zu beanspruchen (sog. Kanonen-' schußbreite), verfügte auch die spanische Kolonie Kuba über eine derartige Sicherheitszone. 1863 wurde dann durch ein königliches Dekret bestimmt, daß das Küstenmeer, in Anbetracht der piratistischen und freibeuterischen Bedrohung, gegen die Proteste der USA auf eine Breite von $6 \mathrm{sm}$ ausgedehnt werden sollte. Diese Bestimmung wurde vorübergehend während der militärischen Besetzung der Insel durch die USA im Jahre 1901 wieder rückgängig gemacht: Die Breite des Küstenmeeres wurde zunächst auf $4 \mathrm{sm}$, nach der formalen Unabhängigkeit Kubas wieder auf $3 \mathrm{sm}$ reduziert.

Im Jahre 1915 richtete Kuba eine 12 sm breite Zoll- und Steuerhoheitszone ein und wich damit zum ersten Mal vom Konzept der reinen Verteidigungs- und Sicherheitsfunktion des Küstenmeeres ab. 1934 wurden alle Gewässer zwischen der Küstenlinie der Hauptinsel und den anliegenden Inseln und Riffen zu internen Gewässern erklärt, in denen ausschließlich die Republik Kuba über das Recht auf freie Schiffahrt, Fischerei und Nutzung anderer Meeresschätze verfügen sollte. Die Küstenmeerzone selbst wurde erst ab der äußeren "nationalen Küstenlinie" gemessen, die vorhandene Flußmündungen, Hafenanlagen, Inseln und Riffe durch gerade Linien verbindet. Ein Jahr nach der Truman-Proklamation über den kontinentalen Schelf der USA wurde 1946 die submarine Plattform Kubas bis zu einer Tiefe von 200 brazas $(1$ braza $=1,6718 \mathrm{~m})$ ins nationale Territorium einbezogen. 3

In Artikel 10 der kubanischen Verfassung von 1976, also 18 Jahre nach der Revolution, wurde erstmals neben der Aufzählung der Inseln, die zum kubanischen Territorium gehören, auch eine Küstenmeerzone und die darüberliegende Luftsäule unter vollständige nationale Souveränität gestellt. 4

3 Nuñez Jiménez, A., El Archipiélago - Cuba: La Naturaleza y el Hombre. La Habana 1982, S. 123 f.

4 "Articulo 10. El Estado socialista cubano ejerce su soberanía: a) sobre todo el territorio nacional, integrado por la Isla de Cuba, la Isla de Pinos, las demás islas y cayos adyacentes, las aguas interiores, el mar territorial en la extensión que fija la Ley y el espacio aéreo que sobre los mismos extiende, b) sobre los recursos naturales y vivos del lecho y subsuelo marinos, y los existentes en las aguas suprayacentes inmediatas a las costas fuera del mar territorial en la extensión que fija la Ley, conforme la práctica intemacional." Nuñez Jiménez 1982, S. 125. 
Die in der Verfassung geforderte gesetzliche Verankerung der Breite der territorialen See erfolgte 1977 mit der Gesetzesverordnung Nr. 1. Darin wurde in Art. 1 eine $12 \mathrm{sm}$ breite Küstenmeerzone ausgewiesen, die von einer geraden Basislinie gemessen wird, wie sie schon im Genfer Übereinkommen über das Küstenmeer und die Anschlußzone von 1958 jedem Staat zugestanden wird, der über besonders zerklüftete Küsten oder der Küste vorgelagerte Inseln und Riffe verfügt (Art. 4 ff. des Ưbereinkommens). Diese Basislinie wurde in Art. 2 der Gesetzesverordnung Nr. 1 mit einer Gesamtlänge von 1380,65 sm, die alle äußersten Punkte der Küste, der vorgelagerten Inseln und Riffe verbindet, und durch die Koordinaten von 124 Wendepunkten genau festgelegt (s. Abb. 1).5 Art. 3 bestimmt im Einklang mit Art. 5 des Genfer Übereinkommens, daß alle landwärtigen Gewässer ab der Basislinie als Binnengewässer gelten, und Art. 4 erklärt, da $\beta$ alle lebenden und nicht lebenden Ressourcen des Meeres, des Meeresbodens und des Luftraumes in und über den Binnengewässem und der Küstenzone unter volle nationale Souveränität gestellt werden. Die Gesetzesverordnung Nr. 2 von 1977 bestimmt die Ausweisung einer 200 sm breiten EEZ, die ebenfalls ab der Basislinie gemessen wird und die dem kubanischen Staat das Recht auf Abbau, Ausbeutung und Erhaltung lebender und nicht lebender natürlicher Ressourcen sichert, die sich im und auf dem Meeresboden und in der Wassersäule befinden.

Fremden Staaten steht das Recht auf freie Seeschiffahrt, freien Überflug und die Verlegung unterseeischer Kabel zu. Eventuelle Ansprüche angrenzender Staaten auf eine eigene Wirtschaftszone werden vom kubanischen Staat respektiert, und Überschneidungen sollen durch bilaterale Abkommen geklärt werden. 6 Mit diesen Gesetzen wurden alle für Kuba relevanten Teile der neuen Konvention Verfassungswirklichkeit, zu einer Zeit, in der die Verhandlungen über die Schelfabgrenzung und über das Tiefseebodenregime noch gar nicht abgeschlossen waren.

Da direkte Nachbarn teilweise näher als 400 sm liegen und Kuba nur über eine relativ kleine submarine Plattform verfügt, wird eine Ausweitung des Schelfbereiches über die $200 \mathrm{sm}$ breite EEZ hinaus für Kuba unmöglich. Auf eine Ausweisung einer $12 \mathrm{sm}$ breite Anschlußzone wird verzichtet. Die theoretisch mögliche Reklamierung eines Archipelstatus wird durch die Einrichtung der geraden Basislinie obsolet, außerdem erfüllt Kuba nicht die Bedingung, daß das Verhältnis Wasser : Land zwischen 1:1 und 9:1 liegen muß7. Damit wird die kubanische Unterzeichnung der Seerechtskonvention von 1982 und ihre Ratifizie-

5 Vgl. hierzu Cuba - Straight Baseline in: Office of the Geographer - Bureau of Intelligence and Research (Hrsg.): Limits in the Seas. Washington, D.C., 28.10.1977, No. 76.

6 Nordquist, M. (Hrsg.): New Directions in the Law of the Sea. Documents - Vol. VII, VIII, IX und X. London 1980, S. 385 f.

7 United Nations (Hrsg.): The Law of the Sea - United Nations Convention on the Law of the Sea. New York 1983, S. 15. 


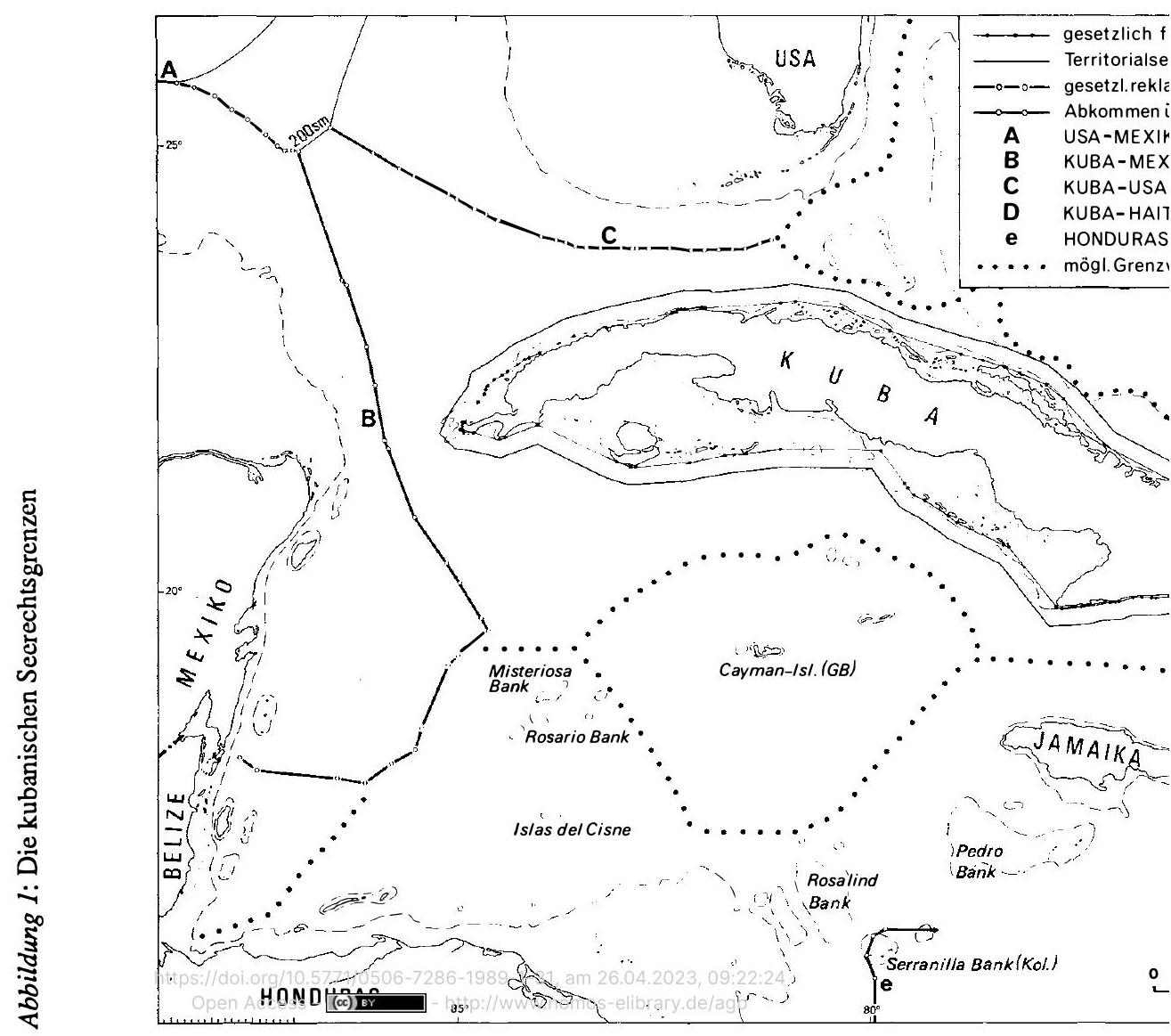


rung am 15. August $1984^{8}$ nur noch zur nachträglichen Bestätigung der ex ante veränderten Verfassungswirklichkeit.

\section{Abkommen und Konflikte mit den Nachbaarstaaten}

Die in Verbindung mit der Ausweitung der Seerechtszonen stehende "territoriale" Vergrößerung Kubas führte unwillkürlich zu einer Uberlagerung der seerechtlichen Ansprüche der sehr nahe liegenden Nachbarstaaten, die ihrerseits ebenfalls ausgedehntere Seerechtsansprüche geltend machen. So haben im Verlaufe der Verhandlungen über die UNSeerechtskonvention von 1982 alle kubanischen Nachbarstaaten, die USA im Norden, die Bahamas im Nordosten, Haiti im Osten, die Cayman-Islands, Jamaika und Honduras im Süden und Mexiko im Westen, entweder eine $200 \mathrm{sm}$ breite Fischereizone oder eine $200 \mathrm{sm}$ breite EEZ eingerichtet. Allein der südliche Nachbar Jamaika hat lange auf die Einrichtung einer solchen Zone verzichtet, plant aber ein Gesetz über die Ausweisung einer $200 \mathrm{sm}$ breiten ausschließlichen Wirtschaftszone. Im Bewußtsein dessen wurde schon bei der Proklamation der kubanischen EEZ darauf hingewiesen, daß etwaige gleiche Rechte anliegender Staaten auf eine Wirtschaftszone respektiert werden, und die Bereitschaft erklärt, bilaterale Abkommen zu schließen, sofern einseitige Proklamationen über nationale Seerechtszonen zu Konflikten führen.

Das erste dahingehende Abkommen wurde 1976, noch vor der Ausweisung der kubanischen ausschließlichen Wirtschaftszone, mit Mexiko geschlossen. Beide Staaten einigten sich auf der Grundlage des Äquidistanzprinzips über eine Trennungslinie durch den Yukatankanal, der das Karibische Meer mit dem Golf von Mexiko verbindet. Diese Linie, die durch 13 Wendepunkte bestimmt wird, trennt neben den beiden Wirtschaftszonen auch den Bereich des Kontinentalschelfes. 9

Das zweite Abkommen wurde im Oktober 1977 mit der Republik Haiti geschlossen. Auch hier fand eine Abgrenzung der Wirtschaftszonen und des Kontinentalschelfes mit Hilfe des Äquidistanzprinzips und durch die Ausweisung einer Linie mit 51 Wendepunkten statt. Beide Staaten erklärten sich bereit, auf jegliche Ansprüche auf Souveränität oder Jurisdiktion im Bereich des anderen Staates zu verzichten. 10 Während der Amtszeit von Präsident Carter in den USA war es sogar möglich, einen Vertrag über die Abgrenzung des Meeres zwischen den seit der kubanischen Revolution verfeindeten Staaten Kuba und den USA zu unterzeichnen, obwohl die Streitfrage des US-Militärstützpunktes Guantánamo11, der nach

8 Die kubanische Ratifizierung der UN-Konvention enthält den Vorbehalt, daß der kubanische Staat die Jurisdiktion des noch einzurichtenden Internationalen Seegerichtshof es nicht anerkennen wird.

9 Maritime Boundary: Cuba - Mexico in: Office of the Geographer - Bureau of Intelligence and Research (Hrsg.): Limits in the Seas. Washington, D.C., 10.9.1985, No. 104.

10 Nordquist 1980, S. 69 f.

11 Der Militärstützpunkt Guantánamo, der die einzige Landgrenze im Inselstaat Kuba darstellt, geht auf die US-amerikanische Besatzungszeit in Kuba zu Beginn dieses Jahrhunderts zurück. In 
Ansicht Kubas widerrechtlich besetzt wird, bis heute nicht geklärt ist. Im Abkommen von 1977 zwischen Kuba und den USA wurde durch die Festlegung einer Trennlinie mit 27 Wendepunkten die Floridastraße in einen US-amerikanischen und einen kubanischen Teil getrennt (s. Abb. 1). Keiner der beiden Staaten soll jenseits seiner Grenzlinie souveräne Hoheitsrechte über das Wasser, den Meeresboden oder den Meeresuntergrund beanspruchen oder ausüben. 12

Die einzigen offenen Streitigkeiten wurden bislang mit den Bahamas ausgetragen, nachdem zwei kubanische Fischerboote wegen der Verletzung der Fischereigrenze von den Bahamas aufgebracht wurden und daraufhin ein bahamaisches Küstenschutzboot durch kubanische Jagdbomber versenkt wurde.13 Ein bilaterales Abkommen über die genaue Abgrenzung der beiden Hoheitsbereiche gibt es bislang nicht. Die in Abb. 1 angegebene Seerechtsgrenze ist entsprechend den Grenzen zu den Cayman-Islands, Honduras und zu Jamaika, mit denen auch keine Grenzabkommen existieren, eine auf dem Äquidistanzprinzip basierende mögliche Kompromißlösung.

So fältt auf, daß gerade zwischen allen drei Archipelstaaten und -territorien, die entweder unter britischer Kolonialmacht standen oder noch stehen14, kein Abkommen mit Kuba existiert, und daß die drei anderen Staaten, jeweils an einer wichtigen Passage des Karibischen Raumes gelegen, über ein Abkommen die Grenzfragen regeln konnten.

\section{Das "neue" Kuba im Kartenbild}

Sobald man dieser neuen Situation nicht nur in seerechtlichen Abhandlungen, sondern auch in allgemein üblichen Kartenwerken, die allzuoft auf eine Darstellung von Seegrenzen verzichten, Rechnung trägt, wird der Eindruck von Kuba als langgestreckter Insel im "blauen Meer" doch erheblich modifiziert.

Nach Festlegung der geraden Basislinie, die alle zum Staat gehörenden Cayos und Inseln ins Territorium integriert, nach Ausweisung des $12 \mathrm{sm}$ breiten Küstenmeeres, in der volle nationale Souveränität herrscht, nach Festlegung einer $200 \mathrm{sm}$ breiten ausschließlichen

Zusammenhang mit dem Platt-Amendment sicherten sich die Vereinigten Staaten das Recht auf Intervention bei politischen Krisensituationen und die Errichtung eines Militärstützpunktes auf Kuba. Trotz massiver Proteste der nachrevolutionären Regierung in Kuba und einer einseitigen Kündigung des Pachtvertrages sind die USA nicht bereit, diese Militärbasis aufzugeben. Vgl. hierzu Toste Ballart, G., Guantánamo: U.S.A. al desnudo. La Habana 1983. In amtlichen kubanischen Kartenwerken wird dieser Stützpunkt nicht berücksichtigt, ebensowenig bei der Ausweisung der kubanischen "straight baseline", die für die Berechnung der Seerechtszonen von großer Bedeutung ist. So kommt es im Bereich der Bucht von Guantánamo zu Überschneidungen der kubanischen und US-amerikanischen Ansprüche.

12 Nordquist 1980, S. 66 f.

13 Hamburger Abendblatt 1980, Nr. 111, S. 14.

14 Die Bahamas wurden 1973 und Jamaika 1962 unabhängig. Die Cayman-Islands sind Kronkolonie mit beschränkter innerer Selbstverwaltung. 
Wirtschaftszone, in der dem Staat eingeschränkte souveräne Rechte zustehen, und nach Abschluß der drei genannten Grenzverträge wird die Gestalt des Staatsgebiets kompakter. Durch die Integration der über die Hauptinsel hinausgehenden Bereiche, nicht allein auf rechtspolitischer, sondem auch auf kognitiver Ebene, kommt man demnach zu einem neuen territorialen Selbstverständnis 15, und die Grenze der EEZ wird in Zukunft die Küstenlinie als geopolitische Basislinie der Meeres- und Weltherrschaftskarte ablösen.

In Abb. 2 wird durch die überspitzte Darstellung der Souveränitätsbereiche, mit vollständiger Souveränität bis zur Grenze der territorialen See und eingeschränkter Souveränität in der EEZ, und das bewußte Unterdrücken der Küstenlinie das wahre Territorium des Staates Kuba veranschaulicht, das neben der gesamten Landfläche von 114.500 qkm noch zusätzlich 248.300 qkm Meeresfläche umfaßt.

\section{Die Fischereipolitik Kubas}

Da die Tragweite der Grenzveränderungen im Meeresraum nicht allein auf die Vergrößerung des Staatsterritoriums und die damit in Zusammenhang stehenden Grenzüberschneidungen beschränkt bleibt, müssen neben den territorialen Auswirkungen auch die wirtschaftlichen Veränderungen nach Ausweitung der Seerechtszonen berücksichtigt werden. In erster Linie sind dabei die Fischereigewohnheiten eines Staates gemeint, die nicht nur durch die Erweiterung der eigenen Fischereizone verändert werden, sondern ebenso durch eine nationale Appropriierung von Teilen der Hohen See, die bislang freiverfügbare Femfischfanggründe waren, betroffen sind. In zweiter Linie gehören dazu die möglicherweise im oder auf dem Bereich des kontinentalen Schelfes befindlichen nicht lebenden Ressourcen, wie Kohlenstoff verbindungen und Erze, die einen bisweilen unbekannten Beitrag zur Wirtschaftskraft eines Staates leisten können.

Im Falle Kubas ist allein die Fischereipolitik von Interesse, da abgesehen von spärlichen Ölvorkommen vor der Nordküste keine weiteren Ressourcenprospektionen vorhanden sind. Traditionell findet eine Nutzung des Fischreichtums im karibischen Raum nur sehr unzureichend statt. So wurde z.B. im vorrevolutionären Kuba der größte Teil der benötigten Fische und Fischkonserven importiert. Erst nach 1959 wurden die wenigen Fischer kooperativ organisiert und die kubanische Fischfangflotte von Grund auf modemisiert, wobei mehrere Fischereihäfen und Werften erweitert oder neu gebaut wurden. Schließlich wurde durch die Einrichtung des Instituto de la Pesca und mehrerer Fischereischulen die Entwicklung der kubanischen Fischereiwirtschaft forciert. Die kubanischen Fischer, die sich vor der Revolution auf die Küstengewässer der Insel beschränkten, begannen mit Hilfe der Regierung mit dem Aufbau einer Fernfangflotte, die neben den Fanggebieten im Golf von Mexiko und des südlichen karibischen Meeres auch neue Fischgründe vor Südwest-Afrika,

15 "... Cuba es más que una isla: es un archipiélago, ... comprende también, la plataforma insular sumergida sobre la que sobresalen sus costas." Vgl. Nuñez Jiménez 1982, S. 65. 


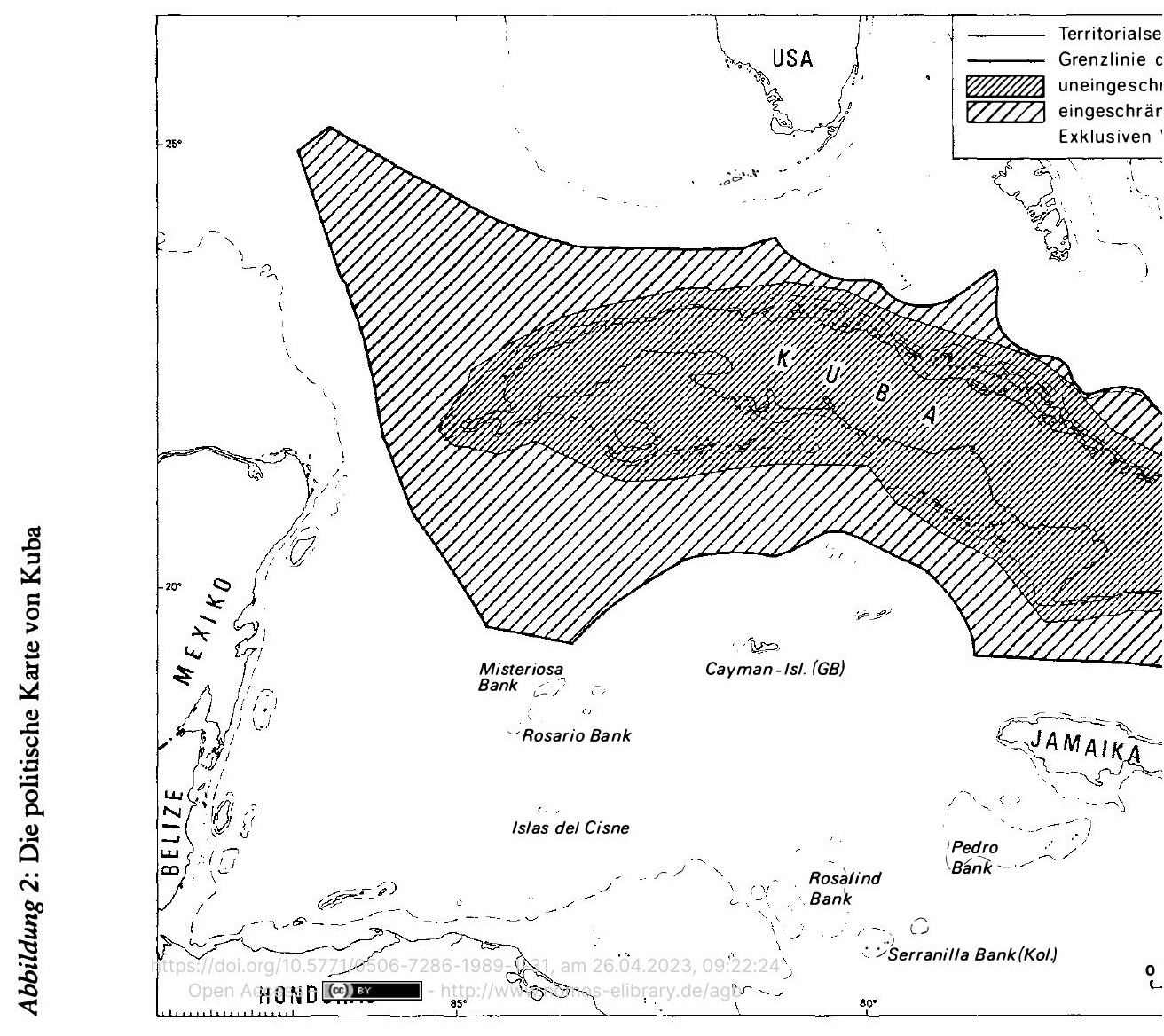


im Südost-Pazifik und im Nord-Atlantik aufsuchten. Zu diesem Zweck wurden drei unterschiedliche Flottenverbände, die Flota Atunera, die Flota del Golfo und die Flota Cubana de Pesca, eingerichtet, die sich mit spezieller Ausrüstung den ihnen zugewiesenen Fischgründen widmen. Die Zunahme der Fischanlandungen von 22.000 (1958) auf 213.200 Tonnen (1978) ist demnach vor allem auf die Erweiterung kubanischer Fischereiaktivitäten im Femfischfang zurückzuführen.16 Bis 1978 hatte es die kubanische Regierung geschafft, mit dem neuen Schwerpunkt Fischereiförderung einen konstanten Zuwachs der Fischanlandungen zu erreichen und zu einer der wichtigsten Fischereinationen Lateinamerikas zu werden. Dem Wirtschaftsbericht der Lloyds Bank Group 17 zufolge rangiert der Exporterlös der kubanischen Fischereiwirtschaft an dritter Stelle nach dem Erlös aus dem Zucker- und dem Nickelexport.

Der Einschnitt in der positiven Entwicklung kubanischer Fischereiwirtschaft kam im Jahr 1979 (s. Tab. 1), wo plötzlich nur noch $72 \%$ der Fangmenge vom Vorjahr erreicht wurden. Der Grund dafür ist vor allem in den Auswirkungen der Seerechtsdiskussion zu suchen, die dazu geführt hat, daß Hochseefischgründe, in denen bislang kubanische Fischerboote aktiv waren, zu ausschließlichen Wirtschaftszonen der angrenzenden Staaten wurden.

Tabelle 1: Die Entwicklung der kubanischen Fischereierträge 1948 - 1982

\begin{tabular}{|c|c|c|c|c|c|c|c|}
\hline \multicolumn{8}{|c|}{ Fangmengen pro Fanggebiet in $1.000 \mathrm{t}$} \\
\hline Jahr & Gesamt & $\begin{array}{l}\text { Nord- } \\
\text { West- } \\
\text { At lantik }\end{array}$ & $\begin{array}{l}\text { West- } \\
\text { Zentral- } \\
\text { At lantik }\end{array}$ & $\begin{array}{l}\text { Ost- } \\
\text { Zentral- } \\
\text { At lantik }\end{array}$ & $\begin{array}{l}\text { Süd-West } \\
\text { At lantik }\end{array}$ & $\begin{array}{l}\text { Suld-Ost } \\
\text { Pazifik }\end{array}$ & $\begin{array}{l}\text { Binnen- } \\
\text { gewässer }\end{array}$ \\
\hline \begin{tabular}{|l|}
1948 \\
1958 \\
1966 \\
1968 \\
1970 \\
1971 \\
1972 \\
1973 \\
1974 \\
1975 \\
1976 \\
1977 \\
1978 \\
1979 \\
1980 \\
1981 \\
1982
\end{tabular} & $\begin{array}{r}8,3 \\
21,9 \\
44,3 \\
66,0 \\
105,9 \\
126,2 \\
139,8 \\
150,3 \\
165,0 \\
143,6 \\
194,1 \\
189,2 \\
213,2 \\
153,9 \\
186,4 \\
164,5 \\
195,3\end{array}$ & $\begin{array}{r}- \\
- \\
1,2 \\
1,2 \\
- \\
1,2 \\
2,0 \\
1,2 \\
- \\
7,5 \\
29,9 \\
18,3 \\
13,1 \\
12,5 \\
9,2 \\
6,7 \\
18,6\end{array}$ & $\begin{array}{c}- \\
- \\
42,7 \\
62,2 \\
61,9 \\
68,0 \\
72,5 \\
72,9 \\
73,1 \\
56,9 \\
77,4 \\
70,6 \\
71,2 \\
66,2 \\
68,4 \\
59,8 \\
68,5\end{array}$ & $\begin{array}{r}- \\
- \\
- \\
0,2 \\
22,0 \\
9,0 \\
7,0 \\
10,6 \\
10,7 \\
6,7 \\
6,4 \\
20,7 \\
7,1 \\
7,5 \\
9,3 \\
8,7 \\
7,1\end{array}$ & $\begin{array}{r}- \\
- \\
0,4 \\
2,1 \\
23,4 \\
46,0 \\
57,3 \\
54,2 \\
53,6 \\
44,6 \\
33,2 \\
33,2 \\
63,5 \\
42,9 \\
4,6 \\
- \\
0,7\end{array}$ & $\begin{array}{c}- \\
- \\
- \\
- \\
- \\
1,2 \\
- \\
10,3 \\
25,5 \\
23,7 \\
43,7 \\
42,8 \\
55,0 \\
19,5 \\
88,6 \\
79,0 \\
86,8\end{array}$ & $\begin{array}{r}- \\
- \\
- \\
0,3 \\
0,6 \\
0,8 \\
1,0 \\
1,1 \\
2,1 \\
4,2 \\
3,5 \\
3,6 \\
3,3 \\
5,3 \\
6,3 \\
10,3 \\
13,6\end{array}$ \\
\hline
\end{tabular}

Quelle: Mena Millar 1985; bfai 1982

16 Bundesstelle für Außenhandelsinfonnation (bfai) (Hrsg.), Kuba: Fischereiwirtschaft - Lage und Entwicklung. Köln 1982, S. 3.

17 Lloyds Bank Group (Hrsg.): Economic Report 1984: Cuba. London 1984, S. 10. 
In Verbindung mit den zunehmenden Input-Kosten, wie hohe Dieselpreise für die Schiffe und steigende Preise für Maschinen und Ausrüstung und mit den zu erwartenden Lizenzzahlungen für Fischereirechte in fremden Wirtschaftszonen, wurde Kuba zu einer strategischen Kursänderung in der Fischereiwirtschaft gezwungen. So mußten zum Beispiel die für den Exporterlös sehr wichtigen Krabbenfischer 18 , die traditionell nicht nur in kubanischen Gewässem, sondem auch vor den Küsten von Mexiko, den USA und Guyana fischten, ihre außerkubanischen Aktivitäten im Zuge der neuen Meeresregime weitgehend einstellen. Ihre Flotte wurde vom Höchststand mit 386 Schiffen (1977) auf 242 Schiffe (1982) reduziert, die nun vor allem in der kubanischen Wirtschaftszone tätig sind. Die damit verbundenen wirtschaftlichen Auswirkungen sind als irreparabel zu bezeichnen. 19 Ähnlich erging es den Ozeantrawlern, die im Atlantik und Südost-Pazifik operierten und durch die Ausdehnung der dortigen Wirtschaftszonen dazu veranlaßt wurden, sich entweder auf Hochseegebiete ohne nationale Jurisdiktion zurückzuziehen oder sich auf Wirtschaftszonen von Staaten zu beschränken, mit denen Kuba ein Fischereiabkommen geschlossen hat. So wurde z.B. am 28. April 1977 ein Abkommen mit den USA unterzeichnet, das den Kubanem nicht nur erlaubt, die von den USA nicht genutzten Fischbestände in der US-Wirtschaftszone auszubeuten, sondem auch die Möglichkeit einräumt, daß kubanische und amerikanische Schiffe zu Versorgungszwecken gegenseitig bestimmte Häfen anlaufen können.20 Weitere Abkommen gibt es mit Angola und Kanada, und in einem Abkommen vom November 1983 mit Mexiko wird Kuba gestattet, in mexikanischen territorialen Gewässem eine jährliche Fangmenge von 3.000 t einzubringen.21

Der durch die Ausdehnung der nationalen Fischereizonen verursachte Ertragsverlust für Kuba, dessen Fangertrag zu zwei Dritteln von der Fernfischerei abhängig war, zwang die Regierung, über die Fangabkommen mit anderen Staaten hinaus zu weiteren Veränderungen in ihrer Fischereiwirtschaft. Kuba entwickelte vier staatliche Ziele, die helfen sollen, die Einbußen möglichst gering zu halten. So sollen erstens die in kubanischen Gewässern befindlichen Fischressourcen maximal genutzt und zweitens die Fangergebnisse der Hochseefischerei auf einem akzeptablen Niveau gehalten werden. Daneben soll drittens die Verarbeitung des Fanges optimiert werden, um bessere Ausgangsbedingungen für den Exporthandel zu erreichen, und die Kosten für Reparaturen an Schiffen und den Bau von neuen Schiffen sollen durch Eigeninitiative möglichst weit gesenkt werden. Viertes Ziel ist es, durch die Entwicklung einer Süßwasserfischzucht in künstlich angelegten Becken oder in den schon vorhandenen Bewässerungsanlagen eine wichtige Alternative zu den verlorenge-

18 Rund $90 \%$ der Crustaceen werden gegen harte Devisen an westliche Industriestaaten verkauft.

19 Mena Millar, A.: Study on the Economic Situation of the Cuban Fisheries, in: Cleveland, B. C. (Hrsg.): Adjustments to Changes in Fisheries Law and Economics. Rom 1985, S. 27-42.

20 Nordquist 1980, S. 250 f. Nach einer Vorlauf phase von zwei Jahren sollte dieses Abkommen nach Ratifizierung und Austausch der Unterlagen für immer gültig bleiben, jedoch kam es aufgrund der unerwarteten Verschlechterung der Beziehungen zwischen beiden Ländem 1979-1980 niemals zu einer Implementierung.

21 Lloyds 1984, S. 10. 
gangenen Fanggründen zu schaffen. Erste Erfolge dieser Initiative mündeten in einer Süßwasserfischproduktion von $11.728 \mathrm{t}$ (1982) (s. Tab. 2), die als sehr beachtlich gewertet werden muß, denn weder der Fang noch der Verzehr dieser Fische kann als Gewohnheit auf Kuba bezeichnet werden. 22

Tabelle 2: Prod.- und Fangqualitäten der kubanischen Fischindustrie 1975 - 1985

\begin{tabular}{|l|c|c|c|c|}
\hline \multicolumn{5}{|c|}{ Fangmengen der Fischerei in $t$} \\
\hline \multicolumn{4}{|c|}{ Art des Fanges } \\
\cline { 2 - 5 } Jahr & insgesamt & \multicolumn{4}{|c|}{ däßon: } \\
\hline 1975 & 143.325 & 900 & 116.474 & 20.000 \\
1978 & 213.170 & 2.200 & 182.235 & 20.916 \\
1979 & 153.799 & 4.300 & 122.065 & 19.017 \\
1980 & 186.481 & 5.737 & 158.127 & 17.233 \\
1981 & 164.815 & 8.822 & 132.472 & 16.888 \\
1982 & 195.246 & 11.728 & 158.177 & 19.203 \\
1983 & 198.394 & 12.677 & 162.214 & 17.402 \\
1984 & 199.623 & 14.529 & 159.384 & 19.211 \\
1985 & 219.884 & 15.473 & 172.921 & 20.683 \\
\hline
\end{tabular}

Quelle: Statistisches Bundesamt 1985, 1988

Zur Erreichung des ersten Zieles, der maximalen Nutzung der kubanischen Fischvorkommen wurde eine Reorganisation der Küstenfischerei, wie die Entwicklung der Fischerei im Schelfbereich und in den Tiefmeerzonen rund um Kuba, vorgenommen. Zu diesem Zweck wurden vier Hauptfischfangzonen eingerichtet (s. Abb. 3). Für jede Region spezifische Programme sollen zu einer optimalen Nutzung der Fischbestände führen. Einer Untersuchung des Centro de Investigaciones Pesqueras zufolge ist die Zone D mit $60 \%$ der Schiffe die wichtigste Fischereizone im kubanischen Küstenfischfang. Es folgt die Zone A mit $33 \%$ der Schiffe, während in den Zonen B und C diese Art der Fischerei nur eine geringe Rolle spielt.23 In der gesamten EEZ Kubas liegt der jährliche Fischertrag bei rund 65.000 t Fisch, der nach dem geschätzten Potential von 80.000 t nur noch um den relativ geringen Betrag von 15 - $20 \%$ gesteigert werden kann. 24

22 Mena Millar 1985, S. 34.

23 García del Barco, F.; Crespo Leon, I., Descriptión de la Pesquería de Peces en las Areas Costeras y Estuarinas de Cuba, in: Revista Cubana de Investigaciones Pesqueras. Año 6, No. 2 1981, S. 5069. bfai 1984. 


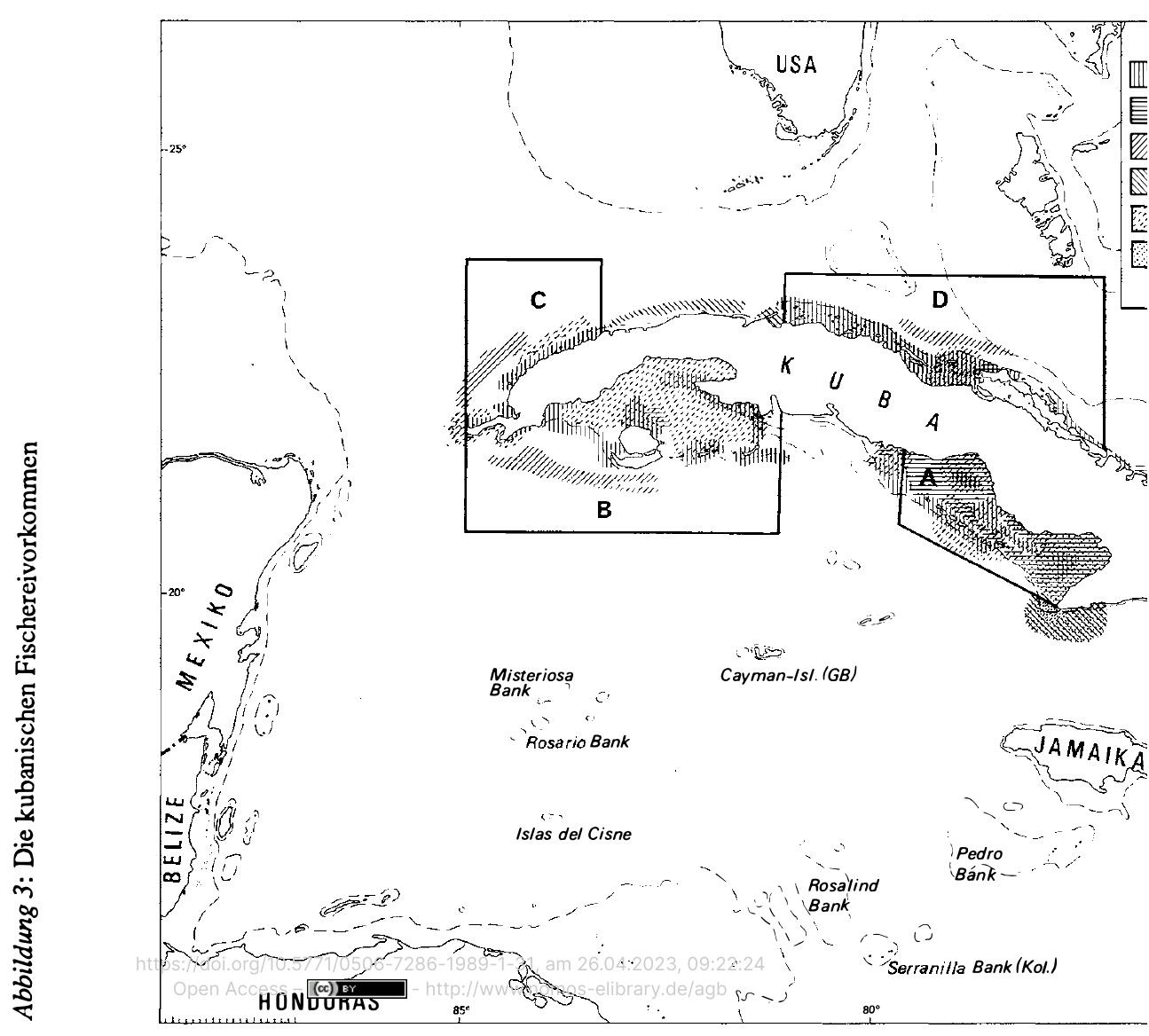


Nach dem Einschnitt in der Fischereientwicklung zum Ende der siebziger Jahre war es dem kubanischen Staat also möglich, mit einer kurzfristigen Kursändenmg in der Strategie, die bis dahin auf große Gewinne aus der Hochseefischerei aufgebaut war, die Einbußen in diesem für die Gesamtwirtschaft sehr wichtigen Teilbereich gering zu halten. Kuba ist es bis 1982 gelungen, die Fangergebnisse zu stabilisieren und wieder ein Niveau von kurz vor 1979 zu erreichen. Damit sind die durch die neue Abgrenzung der Meere entstandenen Nachteile für Kuba mit einer umfassenden Umorientierung auf in eigenen Gewässern befindliche Ressourcen gegenwärtig auf ein Mindestmaß reduziert. Für die Zukunft ist jedoch zu befürchten, daß die Fischvorkommen in eigenen Gewässem nicht mehr ausreichen werden und daß neue Wege gefunden werden müssen, den kubanischen Fischbedarf zu decken.

\section{Schlußbemerkung}

Kubas Beispiel zeigt, daß die nationale Aneignung weiter Meeresteile nicht nur zur Vergrößerung der staatlichen Handlungsbereiche führt, sondern daß die wirtschaftlichen Aktivitäten der einzelnen Staaten direkt durch die neuen Seerechtsbestimmungen beeinflußt werden, obwohl die Konvention noch nicht in Kraft ist. Kuba wurde in diesem Zusammenhang zu Kurskorrekturen in seiner Fischereipolitik gezwungen, die positive Ergebnisse gebracht haben.

Es werden sich jedoch im Zusammenhang mit den Veränderungen des neuen internationalen Seerechts in Zukunft verschiedenartige Konfliktfelder zeigen, die weit über die bloße Abgrenzung zwischen zwei bislang nicht benachbarten Staaten hinausgeht. In diesem Zusammenhang steigt parallel zur Bedeutung der Seegrenzen als Abgrenzungs- und Sicherheitszone des Staates die Konfliktträchtigkeit in Gebieten, in denen es durch wachsende Ansprüche der Nachbarstaaten zu Uberlappungen der Staatsgebiete und zu Nutzungskonkurrenzen bei organischen und mineralischen Ressourcen kommen wird. 
factual acts, incurably null and legally void. From a formal point of view, the confirmation would have to be seen as a completely new enactment with retroactive effect. According to the Supreme Court the exclusion of certain statutes was not to be objected to, particularly not for a violation of the principle of legal certainty nor for the protection of vested rights. The author in substance welcomes the decision. His objections point towards the somewhat superficial treatment of problems concerning the rule of law, and the non-consideration of implications for public international law.

\section{The Influence of the New Law of the Sea on Cuba's Maritime Zones and Fishing} Policy

\section{By Beate Ratter}

After the signing of the Convention on the Law of the Sea in Jamaica in 1982 it will be interesting to ask about the effects of this new international instrument on national policies. There is, in fact, considerable influence not only on the legislation of each country but also on their economic development. The Cuban policy, of extending maritime zones and reorganizing the fishing industry, will be an example of the dimension of this impact.

Nearly all the regulations of the new convention are part of the Cuban legislation, i.e. the Straight Baseline, $12 \mathrm{~nm}$ Territorial Sea, and the $200 \mathrm{~nm}$ Exclusive Economic Zone (EEZ). In connection with the extension of the Cuban Territorial Sea and the reclamation of an Exclusive Economic Zone the Cuban government had to negotiate with its neighbours the extent of these limits in the sea. Treaties were signed with Mexico (1976), with Haiti (1977), and with the United States (1977). The frontiers with Honduras, the Cayman Islands, Jamaica and the Bahamas are not yet under negotiation and therefore the delimitation in respect of these countries needs further clarification.

Besides these changes, the new provisions of the Law of the Sea Convention had an essential influence on the fishing industry in Cuba. Formerly the Cuban fleet war used to exploit international fishing grounds in the Southeast Pacific, the North and South Atlantic which now are no longer international waters because of the proclamation of a $200 \mathrm{~nm}$ EEZ in different parts of the world. The affected Cuban fishing industry decided to improve the exploitation of their own fishing grounds around Cuba and finally they were successful in concentrating their activities in their own EEZ. 